\title{
Pourquoi des dispositions de fin de vie?
}

\section{Martin Rothlin *}

Membre du Conseil de fondation de la Fondation suisse de cardiologie, cardiologue durant de longues années au service de chirurgie cardiaque de l'Hôpital universitaire de Zurich, plus tard partenaire fondateur du centre cardiologique Hirslanden à Zurich.
Correspondance:

Prof. Dr Martin Rothlin

Case postale

St. Niklausweg 48

CH-6045 Meggen

m.e.rothlin-meggen@bluewin.ch
La Fondation suisse de cardiologie a élaboré, conjointement avec l'Institut «Dialog Ethik», des dispositions de fin de vie sous la forme d'un formulaire intitulé «DocumentHumain», qui donne une directive explicite sur la réanimation éventuelle du patient en cas de mort subite par arrêt cardiaque. Les raisons qui ont conduit la Fondation suisse de cardiologie à prendre cette décision sont exposées dans les lignes qui suivent.

Ces dernières décennies, les technologies biomédicales ont tellement élargi les moyens de maintenir une personne en vie qu'aujourd'hui, près de la moitié des décès ne survient qu'après renonciation aux mesures prolongeant la vie ou qu'après leur arrêt. De ce fait, près d'un décès sur deux est consécutif à une décision médicale. On estime communément que cette décision revient au médecin responsable, bien que dans la plupart des cas, celui-ci n'ait pas consulté la personne concernée ou ses proches pour connaître sa volonté. Ce mode de faire correspond plus au paternalisme médical d'autrefois qu'à l'autonomie des patients recherchée aujourd'hui. Si les gens étaient mieux informés à ce sujet, un nombre bien plus important y réfléchiraient et indiqueraient comment ils souhaitent terminer leur vie. Le taux, actuellement de $10 \%$, de personnes admises à l'hôpital avec des dispositions de fin de vie augmenterait à coup sûr.

Pour les jeunes patients, le maintien en vie est pratiquement toujours une priorité. Passé l'âge de 65 à 75 ans, les valeurs sont différentes: pour beaucoup, la qualité de vie l'emporte nettement sur la durée de vie. Ce qui ne signifie pas que ces personnes ont envie de mourir, mais qu'elles sont disposées à réfléchir à la finitude de l'existence et à exprimer leur volonté quant à leur fin de vie. En notre qualité de médecin, nous devrions nous entretenir à ce sujet avec les personnes concernées: la plupart d'entre elles nous en seraient reconnaissantes.

Cependant, il est impossible de tout prévoir pour chaque situation en particulier. On peut également imaginer qu'une personne prenne, au moment crucial, une décision contraire à ses dispositions initiales et place alors les responsables face à un dilemme. Mais sonder la volonté supposée du patient peut aussi être une tâche très lourde pour le personnel soignant qui ne parvient de loin pas toujours à la détecter, comme le révèle une étude faite à ce sujet [1].

\section{Quand les dispositions de fin de vie sont-elles importantes?}

Le formulaire de dispositions de fin de vie peut déjà servir lors d'un entretien avec les proches ou avec le médecin traitant, comme base de discussion pour cerner les problèmes importants et parvenir à une décision quand la situation s'y prête. Bien des personnes pensent qu'il suffit «d'avoir parlé de tout» avec leur médecin de famille ou une autre personne de confiance. Mais quand l'hospitalisation devient nécessaire, le médecin de famille n'accompagne pas son patient. A l'hôpital justement, où le patient rencontre des médecins qui lui sont complètement étrangers, il serait particulièrement important qu'il puisse transmettre à l'équipe soignante des voux clairement exprimés dans des dispositions de fin de vie accessibles 24 h sur 24, à l'exemple du «DocumentHumain». N'oublions pas le cas du patient qui n'est plus en mesure d'exprimer ses vœux quand il entre à l'hôpital!

Il est préférable qu'une personne remplisse et dépose ses dispositions de fin de vie avant de tomber gravement malade et d'être éventuellement hospitalisée d'urgence. Il faut en effet parvenir à une décision rationnelle sans subir la pression du temps et surtout sans angoisse.

Pourquoi faut-il des dispositions indiquant explicitement si le patient désire ou non une réanimation en cas d'arrêt cardiaque? Quand un arrêt cardiaque se produit subitement, le patient perd connaissance et ne peut plus s'exprimer. Dans cette situation, les secouristes, ambulanciers ou médecins urgentistes prennent des mesures immédiates en appliquant les règles de leur métier sans mandat spécial. Ils sont habilités à le faire par la volonté supposée de la personne concernée de survivre et par le serment d'Hippocrate qu'ils ont prêté.

Pour agir de la sorte, il faudrait toutefois que la volonté des personnes âgées soit véritablement d'être réanimées dans tous les cas. On peut toutefois supposer que la volonté de renoncer à une réanimation, et donc l'autonomie de nombreuses personnes âgées, n'est pas respectée dans bien des cas. Dans le cadre de l'étude américaine approfondie SUPPORT [2], une enquête a été menée sur la réanimation auprès de patients hospitalisés souffrant d'une grave insuffisance cardiaque. Un tiers des personnes âgées de plus de 75 ans a rejeté l'idée d'une réanimation; ce 
pourcentage était moins élevé chez des personnes plus jeunes, par exemple de $9 \%$ chez les moins de 40 ans. Des enquêtes plus circonscrites réalisées aux Etats-Unis et en Europe ont donné les mêmes résultats. Outre l'âge, d'autres facteurs jouent également un rôle dans la décision en faveur ou non d'une réanimation, tels que la qualité de vie, l'état de santé, l'environnement social, les valeurs personnelles et la conception de l'existence ainsi que la peur d'une future dépendance $[2,3]$.

La plupart des études contenaient des données sur le manque d'information des personnes sondées, mais pas d'intervention par l'information quantitative sur les faibles chances de succès d'une réanimation et les possibles séquelles ultérieures. Or, ces informations devraient être une condition préalable absolue à toute prise de décision réaliste par les personnes concernées, au sens d'un «consentement éclairé». Dans une étude d'intervention [4], des spécialistes en gériatrie ont interrogé des patients ambulatoires sur la réanimation en cas d'arrêt cardiaque dans le cadre d'une maladie aiguë: $41 \%$ ont préféré la réanimation. Après des explications détaillées et compréhensibles sur les perspectives et les complications possibles, la volonté d'être réanimé a baissé à $22 \%$. En Europe, des enquêtes menées auprès de patients ambulatoires ou hospitalisés âgés ont révélé qu'après des informations médi-

\section{«DocumentHumain», dispositions de fin de vie téléchargeables 24 h sur 24}

On peut télécharger gratuitement le formulaire «DocumentHumain» élaboré par la Fondation suisse de cardiologie et l'institut «Dialog Ethik» dans l'internet, sous www. swissheart.ch et www.dialog-ethik.ch, ou le commander comme document imprimé au prix de Fr. 12.- à ces deux organisations. Le «DocumentHumain» une fois rempli est enregistré électroniquement par l'institut «Dialog Ethik» dans le respect de la protection des données. Les imprécisions qui pourraient rendre les dispositions juridiquement non contraignantes sont éclaircies par téléphone avec les personnes concernées. Les dispositions de fin de vie déposées peuvent en tout temps être consultées par une ligne d'assistance téléphonique. Pour I'enregistrement électronique, l'institut «Dialog Ethik» perçoit une contribution aux frais de Fr. 120.-; la mise à jour tous les deux ans (ou en cas de besoin) coûte Fr. 30.- et garantit la validité du document. cales détaillées, $48 \%$ des personnes interrogées à Helsinki [5], 65\% à Leiden [6], 74\% à Dublin [7] et même $75 \%$ à Reykjavik [8] refusaient une réanimation. En Suisse, aucun chiffre significatif n'est malheureusement disponible à ce sujet.

\section{Comment la volonté des personnes âgées pourrait-elle être mieux respectée?}

Des dispositions de fin de vie, à l'instar de celles élaborées par la Fondation suisse de cardiologie avec l'institut «Dialog Ethik», sont une aide précieuse pour les médecins avant de décider de la poursuite des traitements. Pour les cas d'urgence toutefois, les ambulanciers et les médecins urgentistes estiment à bon droit qu'ils ne peuvent perdre aucun instant à rechercher des dispositions de fin de vie ou un document de ce genre. Les Etats-Unis connaissent un système qui fonctionne bien. Des organisations d'utilité publique officiellement reconnues gèrent électroniquement des dispositions de fin de vie en respectant pleinement la protection des données (comme l'institut «Dialog Ethik» le fait en Suisse). Sur demande, elles remettent aux personnes concernées un médaillon avec leur identité, leurs dispositions et, lorsque cela est pertinent, leur volonté concernant une réanimation éventuelle. Ce médaillon peut être porté au cou. Selon une enquête faite dans 49 Etats des USA, $89 \%$ des sauveteurs ou médecins urgentistes respectent
- Fondation suisse de cardiologie, Schwarztorstrasse 18, 3000 Berne 14, tél. 031 38880 80, fax 03138880 88, e-mail: info@swissheart.ch, internet: www.swiss heart.ch

- Institut «Dialog Ethik», Sonneggstrasse 88, 8006 Zürich, tél. 04425242 01, fax 04425242 13, e-mail: info@dialogethik.ch, internet: www.dialog-ethik.ch

\section{Pour le corps médical}

La Fondation suisse de cardiologie met gratuitement à disposition des médecins en cabinet privé et à l'hôpital, sur demande, une petite affiche pour les salles d'attente et de consultation ainsi qu'un présentoir avec des prospectus et des cartes de commande pour le formulaire «DocumentHumain» (jusqu'à épuisement du stock) à la Fondation suisse de cardiologie, Schwarztorstr. 18, 3000 Berne 14, tél. 03138880 80, Fax 03138880 88, e-mail: docu@swiss heart.ch, internet: www.swissheart.ch 
ces données et, si la personne l'a exigé, n'entreprennent aucune mesure de réanimation [9]. Il est temps de donner en Suisse aux personnes qui ne désirent pas être réanimées en cas d'arrêt cardiaque subit, une possibilité semblable d'exprimer leurs vœux. L'institut «Dialog Ethik» et la Fondation suisse de cardiologie examineront la création d'un tel médaillon dans le cadre d'un groupe de travail.

\section{Références}

1 Perron NJ, Morabia A, de Torrenté A. Quality of life of Do-Not-Resuscitate (DNR) patients: How good are physicians in assessing DNR patients' quality of life? Swiss Med Wkly 2002;132:562-5.

2 Krumholz HM, Phillips RS, Hamel MB et al. Resuscitation preferences among patients with severe congestive heart failure. Circulation 1998;98:648-55.

3 Schönwetter RS, Walker RM, Kramer DR, Robinson BE. Resuscitation decision making in the elderly: the value of outcome data. J Gen Intern Med 1993;8:295-300.
4 Murphy DJ, Burrows D, Santilli S, et al. The influence of the probability of survival on patient's preferences regarding cardiopulmonary resuscitation. N Engl J Med 1994;330:545-9.

5 Laakkonen ML, Pitkala KH, Strandberg TE, et al. Older people's reasoning for resuscitation preferences and their role in the decision making process. Resuscitation 2005;65:165-71.

6 van Mil AH, van Klink RC, Huntjens C, et al. Cardiopulmonary resuscitation preferences in Dutch community-dwelling and hospitalised elderly people: an interaction between gender and quality of life. Med Decis Making 2000;20:423-9.

7 O'Keeffe ST, Lavan NJ. Cardiopulmonary resuscitation preferences in the elderly. Eur J Med 1993; 2:33-5.

8 Hansdottir H, Gruman C, Curry L, Judge JO. Preferences for CPR among the elderly: the influence of attitudes and values. Conn Med 2000;64:625-30

9 Marco CA, Schears RM. Prehospital resuscitation practices: A survey of prehospital providers. J Emerg Med 2003;24:101-6. 